\title{
Modified fermion tunneling from higher-dimensional charged AdS black hole in massive gravity
}

\author{
Zhong-Wen Feng ${ }^{1,2, a}{ }_{\mathbb{D}}$, Qun-Chao Ding ${ }^{1,2, \mathrm{~b}}$, Shu-Zheng Yang ${ }^{1,2, \mathrm{c}}$ \\ ${ }^{1}$ Physics and Space Science College, China West Normal University, Nanchong 637009, China \\ 2 Department of Astronomy, China West Normal University, Nanchong 637009, China
}

Received: 31 October 2018 / Accepted: 17 May 2019 / Published online: 27 May 2019

(C) The Author(s) 2019

\begin{abstract}
The tunneling behavior of fermions with halfintegral spin from a higher dimensional charged anti-de Sitter (AdS) black hole in de Rham, Gabadadze and Tolley (dRGT) massive gravity is investigated via a modified HamiltonJacobi equation. The results demonstrate that the modified thermodynamic quantities not only are related to the properties of the higher dimensional charged AdS black hole in dRGT massive gravity but also depend on the parameter $\beta$, the coupling constant $\sigma$ and the mass of emitted particles $m$. In addition, the modified Hawking temperature is higher than the original temperature; hence, the effect of MDR can significantly enhance the evolution of the black hole. Besides, our results can be verified using the modified Stefan-Boltzmann law.
\end{abstract}

\section{Introduction}

Based on the classical viewpoint, black holes were once thought to only absorb objects [1]. However, this changed after Hawking proved that black holes can radiate particles. In the theory of black hole radiation (which we now call it "Hawking radiation theory"), Hawking introduced the quantum mechanism into gravity theory of curved spacetime and demonstrated that black holes can emit particles $[2,3]$. This theory profoundly reveals the connection among quantum theory, gravitation theory, thermodynamics, and statistical physics. One can calculate the temperature of black holes using the Hawking radiation. Currently, the theory of Hawking radiation is considered the most important tool for investigating the thermodynamic properties of black holes.

Apart from the original research method of studying the radiation of black holes, many new methods have been pro-

\footnotetext{
a e-mail: zwfengphy@163.com

b e-mail: qcdingmn@163.com

c e-mail: szyangcwnu@126.com
}

posed in the past 40 years [4-10]. In Refs. [6,7], Kraus, Parikh and Wilczek pointed out that the black hole radiation process can be considered as the quantum tunneling. Therefore, regarding the horizons as the tunneling barrier, one can easily obtain the tunneling rate of the emitted particles and the thermodynamic properties of black holes. Subsequently, Srinivasan and Padmanabhan developed the tunneling method and put forward the Hamilton-Jacobi ansatz, which substantially simplifies the research process and promotes the understanding of black holes [8]. Then, Kerner and Mann used the Hamilton-Jacobi ansatz to discuss fermion tunneling from spherically symmetric black holes $[9,10]$. In Refs. [11,12], Yang et al. successfully derived the HamiltonJacobi equation from the Klein-Gordon equation, the Dirac equation, and the Maxwell equations. According to their works, the Hamilton-Jacobi ansatz can be used to describe the tunneling behavior of particles with spin on the horizons of black holes. Hence, the Hamilton-Jacobi ansatz is an effective approach for studying Hawking radiation. Using the Hamilton-Jacobi ansatz, extensive investigations of many types of black holes have been conducted [13-22].

However, the classical theory of Hawking radiation has some defects [23]. It has been found that the radiation that is derived from classical theory corresponds to a pure thermal state; hence, the black hole would emit all information at the end of evaporation, which would leads to the "information loss paradox". In addition, the singularities of spacetimes would be exposed to the universe since the horizons would disappear. For resolving this paradox, many methods have been proposed. In recent years, many works have claimed that there is a minimum measurable length in nature [24-26]. In Refs. [27,28], Amelino-Camelia showed that the standard energy-momentum dispersion relation must be changed to the modified dispersion relation (MDR) nears the minimum measurable length. Based on the MDR, researchers have developed a new approach for overcoming the problems of the classical theory of Hawking radi- 
ation. In Refs. [29-37], the authors studied the modified thermodynamic properties of black holes via the MDR; according to their results, the MRD has significant effect on the evolution of black holes, namely, it prevents black holes from total evaporation and leads to remnants. Meanwhile, it is believed that the MDR can modify the equation of motion of particles on the event horizon of black holes. In Refs. [38,39], by using the MDR $p_{0}^{2}=p^{2}+m^{2}-$ $\left(\ell_{p} p_{0}\right)^{2 \beta} \boldsymbol{p}^{2}$, which appears in space-time foam Liouvillestring models, Kruglov obtained a modified Dirac equation by fixing $\beta=1$, that is, $\left[\gamma^{\mu} \partial_{\mu}+m-i \ell_{p}\left(\bar{\gamma}^{t} \partial_{t}\right)\left(\bar{\gamma}^{i} \partial_{i}\right)\right] \psi=$ 0 . Subsequently, when assessing $\beta=1$, Yang and his collaborators proposed another modified Dirac equation, namely, $\left[\gamma^{\mu} D_{\mu}+m / \hbar-\sigma \hbar\left(\gamma^{t} D^{t}\right)\left(\gamma^{j} D^{j}\right)\right] \Psi=0$ and a new modified Klein-Gordon equation, that is, $\left(-\partial_{t}^{2}+\partial_{j}^{2}+\right.$ $\left.m^{2}-\sigma^{2} \hbar^{2} \partial_{t}^{2} \partial_{j}^{2}\right)=0$, where $\sigma$ is a very small parameter $[40,41]$. Furthermore, all of those works claimed that the effect of MDR can be detected by higher energy experiments.

Most of the works have been limited to the study of the tunneling behavior of particles with spin 0 or $1 / 2$. However, it should be noted that the black holes radiate particles of both integral spin $(0,1, \cdots)$ and half-integral spin $(1 / 2,3 / 2, \cdots)$. Therefore, in this paper, we discuss the modified tunneling behavior of fermions with half-integral spin. First, we derive the modified Hamilton-Jacobi equation from the RaritaSchwinger equation in curved spacetime. Then, we study the fermion tunneling from a D-dimensional charged AdS black hole in dRGT massive gravity via the modified HamiltonJacobi equation. Finally, the correction for the Hawking temperature of the D-dimensional charged AdS black hole in dRGT massive gravity is obtained.

The remainder of this paper is organized as follows: In Sect. 2, according to a modified Dirac equation and the WKB approximation, we derive the modified HamiltonJacobi equation from the Rarita-Schwinger equation. In Sect. 3, by using the modified Hamilton-Jacobi equation, the modified tunneling rate of a fermion with half-integral spin and the correction for the Hawking temperature of a Ddimensional charged AdS black hole in dRGT massive gravity are obtained. The conclusions of this work are presented in Sect. 4.

\section{Modified Hamilton-Jacobi equation for fermions with half-integral spin}

In this section, we derive the modified Hamilton-Jacobi equation from the Rarita-Schwinger equation. In Ref. [42], the Rarita-Schwinger equation is as follows:

$\left(\bar{\gamma}^{\mu} \partial_{\mu}+\frac{m}{\hbar}\right) \psi_{\alpha_{1} \cdots \alpha_{k}}=0$
The above equation is used to describe the kinematics of fermions with half-integral spin in Minkowski spactime, which satisfies the supplementary conditions $\bar{\gamma}^{\mu} \psi_{\mu \alpha_{2} \cdots \alpha_{k}}=$ $\partial_{\mu} \psi_{\alpha_{2} \cdots \alpha_{\kappa}}^{\mu}=\psi_{\mu \alpha_{3} \cdots \alpha_{\kappa}}^{\mu}=0$, which satisfy the commutation relation $\gamma^{\mu} \gamma^{v}+\gamma^{v} \gamma^{\mu}=2 g^{\mu v} I$. The role of the supplementary conditions is restrict the spin of fermions, for instance, if $\psi_{\alpha_{1} \cdots \alpha_{\kappa}}=\psi$, that is, $\kappa=0$, the supplementary conditions vanish, and Eq. (1) becomes the Dirac equation, which describes the spin-1/2 fermion. However, if $\kappa=1$, term $\psi_{\mu \alpha_{3} \cdots \alpha_{\kappa}}^{\mu}$ vanishes and Eq. (1) reduces to the kinematics equation for fermions with spin 3/2 [42].

The spacetimes around the black holes are extremely curved. Therefore, in order to investigate the tunneling behavior of fermions on the event horizon of a black hole, one must generalize the Rarita-Schwinger equation to curved spacetime. In Refs. [40,41], the Rarita-Schwinger equation in curved spacetime is given by

$\left(\gamma^{\mu} \mathcal{D}_{\mu}+\frac{m}{\hbar}\right) \psi_{\alpha_{1} \cdots \alpha_{\kappa}}=0$

Notably, the covariant derivative $\mathcal{D}_{\mu}$ in Eq. (2) is defined as $\mathcal{D}_{\mu} \equiv \partial_{\mu}+\Omega_{\mu}+i e A_{\mu} / \hbar$ with the spin connection in curved spacetime $\Omega_{\mu}$.

To obtain the modified corrected Hamilton-Jacobi equation for fermions with half-integral spin, we need to construct a deformed Rarita-Schwinger equation in curved spacetime by developing the viewpoints in Refs. [38-41,43]

$$
\begin{aligned}
& {\left[\gamma^{\mu} \mathcal{D}_{\mu}+\frac{m}{\hbar}-\sigma \hbar^{\beta}\left(\sqrt{g^{t t}} \mathcal{D}_{t}\right)^{\beta-1}\left(\gamma^{t} \mathcal{D}_{t}\right)\left(\gamma^{j} \mathcal{D}_{j}\right)\right]} \\
& \cdot \psi_{\alpha_{1} \cdots \alpha_{\kappa}}=0,
\end{aligned}
$$

where parameter $\beta$ is a key characteristic of the magnitude of the effect of MDR, the value of which influences the modified results, and $\sigma$ is an extremely small coupling constant, which leads to the correction term $\sigma \hbar^{\beta}\left(\sqrt{g^{t t}} \mathcal{D}_{t}\right)^{\beta-1}$ $\left(\gamma^{t} \mathcal{D}_{t}\right)\left(\gamma^{j} \mathcal{D}_{j}\right)$ being very small. According to Eq. (3), the wave function $\psi_{\alpha_{1} \cdots \alpha_{\kappa}}$ of a fermion with half-integral spin can be expressed as follows:

$\psi_{\alpha_{1} \cdots \alpha_{\kappa}}=\xi_{\alpha_{1} \cdots \alpha_{\kappa}} \exp (i S / \hbar)$,

where $\xi_{\alpha_{1} \cdots \alpha_{\kappa}}$ and $S$ are matrices and the action of the fermion, respectively [29,43-57]. The angular momentum parameters and the radiation energy parameters of radiation particles are denoted as $\partial_{\varphi} S=j$ and $\partial_{t} S=-\omega$, respectively. Now, substituting Eq. (4) into Eq. (3), and ignoring the higher order term $\mathcal{O}(\hbar)$, yields 


$$
\begin{aligned}
& \left\{i \gamma^{\mu}\left(\partial_{\mu} S+e A_{\mu}\right)+m-\sigma\left[\sqrt{g^{t t}}\left(-i \omega+i e A_{t}\right)\right]^{\beta-1}\right. \\
& \left.\cdot \gamma^{t}\left(\omega-e A_{t}\right) \gamma^{j}\left(\partial_{j} S+e A_{j}\right)\right\} \xi_{\alpha_{1} \cdots \alpha_{\kappa}}=0
\end{aligned}
$$

By considering the relation $\gamma^{\mu}\left(\partial_{\mu} S+e A_{\mu}\right)=$ $-\gamma^{t}\left(\omega-e A_{t}\right)+\gamma^{j}\left(\partial_{j} S+e A_{j}\right)$, the equation above can be rewritten as

$$
\begin{aligned}
& \Gamma^{\mu}\left(\partial_{\mu} S+e A_{\mu}\right) \xi_{\alpha_{1} \cdots \alpha_{\kappa}} \\
& \quad+\left[m+\sigma\left(g^{t t}\right)^{\frac{\beta+1}{2}}\left(-i \omega+i e A_{t}\right)^{\beta+1}\right] \xi_{\alpha_{1} \cdots \alpha_{\kappa}}=0,
\end{aligned}
$$

where $\Gamma^{\mu}=\gamma^{\mu}-\sigma\left(g^{t t}\right)^{\frac{\beta-1}{2}}\left(-i \omega+i e A_{t}\right)^{\beta} \gamma^{t} \gamma^{\mu}$. Multiplying $\Gamma^{v}\left(\partial_{v} S+e A_{v}\right)$ by Eq. (6), the results is

$$
\begin{aligned}
& \Gamma^{\mu} \Gamma^{v} \Delta \xi_{\alpha_{1} \cdots \alpha_{\kappa}}+\left[m+\sigma\left(g^{t t}\right)^{\frac{\beta+1}{2}}\left(-i \omega+i e A_{t}\right)^{\beta+1}\right]^{2} \\
& \cdot \xi_{\alpha_{1} \cdots \alpha_{\kappa}}=0,
\end{aligned}
$$

where $\Gamma^{\mu} \Gamma^{v}=\gamma^{\mu} \gamma^{v}-2 \sigma\left(g^{t t}\right)^{\frac{\beta-1}{2}}\left(-i \omega+i e A_{t}\right)^{\beta} g^{t \mu} \gamma^{v}+$ $\mathcal{O}\left(\sigma^{2}\right)$ and $\Delta=\left(\partial_{\mu} S+e A_{\mu}\right)\left(\partial_{v} S+e A_{v}\right)$. Next, the subscripts $\mu$ and $v$ are interchanged. Then, adding the result with Eq. (7), and multiplying it by $1 / 2$ [41], one yields

$$
\begin{aligned}
& \left\{\frac{\gamma^{v} \gamma^{\mu}+\gamma^{\mu} \gamma^{v}}{2} \Delta-\sigma\left(g^{t t}\right)^{\frac{\beta-1}{2}}\left(-i \omega+i e A_{t}\right)^{\beta}\right. \\
& \quad \cdot\left(g^{\mu t} \gamma^{v}+g^{v t} \gamma^{\mu}\right) \Delta+\left[m+\sigma\left(g^{t t}\right)^{\frac{\beta+1}{2}}\left(-i \omega+i e A_{t}\right)^{\beta+1}\right]^{2} \\
& \left.\quad+\mathcal{O}\left(\sigma^{2}\right)\right\} \xi_{\alpha_{1} \cdots \alpha_{\kappa}}=\left\{g^{\mu v} \Delta+m^{2}+2 \sigma m\left(g^{t t}\right)^{\frac{\beta+1}{2}}\right. \\
& \quad \cdot\left(-i \omega+i e A_{t}\right)^{\beta+1}-2 \sigma\left(g^{t t}\right)^{\frac{\beta-1}{2}}\left(-i \omega+i e A_{t}\right)^{\beta} g^{v t} \gamma^{\mu} \Delta \\
& \left.+\mathcal{O}\left(\sigma^{2}\right)\right\} \xi_{\alpha_{1} \cdots \alpha_{\kappa}}=0 .
\end{aligned}
$$

Moreover, by using the anti-commutation relations of gamma matrices, Eq. (8) can be simplified as follows:

$i \sigma \gamma^{\mu}\left(\partial_{\mu} S+e A_{\mu}\right) \xi_{\alpha_{1} \cdots \alpha_{\kappa}}+\mathcal{M} \xi_{\alpha_{1} \cdots \alpha_{\kappa}}=0$,

where

$$
\mathcal{M}=\frac{g^{\mu v} \Delta+m^{2}+2 \sigma m\left(g^{t t}\right)^{\frac{\beta+1}{2}}\left(-i \omega+i e A_{t}\right)^{\beta+1}}{-2 i\left(g^{t t}\right)^{\frac{\beta-1}{2}}\left(-i \omega+i e A_{t}\right)^{\beta} g^{v t}\left(\partial_{v} S+e A_{v}\right)} .
$$

Next, by multiplying the left side of Eq. (9) by $-i \sigma \gamma^{v}\left(\partial_{v} S\right.$ $\left.+e A_{v}\right)$, then, interchanging the subscripts $\mu$ and $v$, adding the result with Eq. (9) and dividing by 2, the final result is obtained:

$$
\begin{aligned}
& {\left[\frac{g^{\mu v} \Delta+m^{2}+2 \sigma m\left(g^{t t}\right)^{\frac{\beta+1}{2}}\left(-i \omega+i e A_{t}\right)^{\beta+1}}{-2 i\left(g^{t t}\right)^{\frac{\beta-1}{2}}\left(-i \omega+i e A_{t}\right)^{\beta} g^{v t}\left(\partial_{v} S+e A_{v}\right)}\right]^{2}} \\
& +\sigma^{2} g^{\mu v}\left(\partial_{\mu} S+e A_{\mu}\right)\left(\partial_{v} S+e A_{v}\right)=0 .
\end{aligned}
$$

Here, the higher order terms, which are denoted as $\mathcal{O}\left(\sigma^{2}\right)$ are ignored since they are very small, and one obtains

$$
\begin{aligned}
& g^{\mu v}\left(\partial_{\mu} S+e A_{\mu}\right)\left(\partial_{v} S+e A_{v}\right) \\
& \quad+m^{2}+2 \sigma m\left(g^{t t}\right)^{\frac{\beta+1}{2}}\left(-i \omega+i e A_{t}\right)^{\beta+1}=0 .
\end{aligned}
$$

Obviously, Eq. (12) is the modified Hamilton-Jacobi equation for fermions with half-integral spin that was derived from the Rarita-Schwinger equation in curved spacetime. According to previous work, the Hamilton-Jacobi equation can be derived not only from the Rarita-Schwinger equation or the Dirac equation but also from the Klein-Gordon equation, the Maxwell equations, and the gravitational wave equation, among others. Therefore, the Hamilton-Jacobi equation can be used to describe the kinematic properties of particles with any spin. In the next section, we use modified HamiltonJacobi equation to investigate fermion tunneling from the D-dimensional charged AdS black hole in dRGT massive gravity.

\section{Fermion tunneling from the D-dimensional charged AdS black hole in dRGT massive gravity}

In this section, the tunneling behavior of fermions on the horizon of a D-dimensional charged AdS black hole in dRGT massive gravity is calculated. In higher dimensional dRGT massive gravity, the action with a negative cosmological constant and a Maxwell field can be expressed as

$$
\begin{aligned}
I= & \frac{1}{16} \int \mathrm{d}^{D} x \sqrt{-g} \\
& \cdot\left[\mathcal{R}-2 \Lambda-\frac{1}{4} F_{\mu \nu} F^{\mu \nu}+m^{2} \sum_{i}^{n} c_{i} \mathcal{U}_{i}(g, f)\right],
\end{aligned}
$$

where $n \leq D-2$. The parameters $\mathcal{R}, c_{i}$ and $f$ are the scalar curvature, a series of constants and a fixed rank-2 symmetric tensor, respectively. $F_{\mu \nu}$ is the Maxwell field strength, which satisfies the relationship $F_{\mu \nu}=\partial_{\mu} A_{\nu}-\partial_{\nu} A_{\mu}$. The last four terms of the above equation represent the massive potential [58-64]. Meanwhile, when considering the higher dimensional massive gravity is ghost free, the symmetric polynomials of the eigenvalues $\mathcal{U}_{i}$ of the $D \times D$ matrix $\left(\mathcal{K}_{v}^{\mu} \equiv \sqrt{g^{\mu \alpha} f_{\alpha v}}\right)$ are [65-67]

$$
\begin{aligned}
& \mathcal{U}_{1}=[\mathcal{K}], \\
& \mathcal{U}_{2}=[\mathcal{K}]^{2}-\left[\mathcal{K}^{2}\right],
\end{aligned}
$$




$$
\begin{aligned}
\mathcal{U}_{3}= & {[\mathcal{K}]^{3}-3[\mathcal{K}]\left[\mathcal{K}^{2}\right]+2\left[\mathcal{K}^{3}\right], } \\
\mathcal{U}_{4}= & {[\mathcal{K}]^{4}-6\left[\mathcal{K}^{2}\right][\mathcal{K}]^{2}+8\left[\mathcal{K}^{3}\right][\mathcal{K}]+3\left[\mathcal{K}^{2}\right]^{2}-6\left[\mathcal{K}^{4}\right], } \\
\mathcal{U}_{5}= & \frac{1}{60}[\mathcal{K}]^{5}-\frac{1}{6}[\mathcal{K}]^{3}\left[\mathcal{K}^{2}\right]+\frac{1}{3}[\mathcal{K}]^{2}\left[\mathcal{K}^{3}\right]-\frac{1}{3}\left[\mathcal{K}^{2}\right] \\
& \cdot\left[\mathcal{K}^{3}\right]+\frac{1}{4}[\mathcal{K}]\left[\mathcal{K}^{2}\right]^{2}-\frac{1}{2}[\mathcal{K}]\left[\mathcal{K}^{4}\right]+\frac{2}{3}\left[\mathcal{K}^{5}\right], \\
\mathcal{U}_{6}= & \frac{1}{360}[\mathcal{K}]^{6}-\frac{1}{24}[\mathcal{K}]^{4}\left[\mathcal{K}^{2}\right]+\frac{1}{9}[\mathcal{K}]^{3}\left[\mathcal{K}^{3}\right]-\frac{1}{4}[\mathcal{K}]^{2}\left[\mathcal{K}^{4}\right] \\
& +\frac{1}{8}[\mathcal{K}]\left[\mathcal{K}^{2}\right]^{2}-\frac{1}{24}\left[\mathcal{K}^{2}\right]^{3}+\frac{1}{9}\left[\mathcal{K}^{3}\right]^{2}-\frac{1}{3}\left[\mathcal{K}^{3}\right]\left[\mathcal{K}^{2}\right] \\
& \cdot[\mathcal{K}]+\frac{1}{4}\left[\mathcal{K}^{4}\right]\left[\mathcal{K}^{2}\right]+\frac{2}{5}\left[\mathcal{K}^{5}\right][\mathcal{K}]-\frac{1}{3}\left[\mathcal{K}^{6}\right], \\
\mathcal{U}_{7}= & \frac{1}{2520}[\mathcal{K}]^{7}-\frac{1}{240}[\mathcal{K}]^{5}\left[\mathcal{K}^{2}\right]+\frac{1}{72}[\mathcal{K}]^{4}\left[\mathcal{K}^{3}\right] \\
& -\frac{1}{24}[\mathcal{K}]^{3}\left[\mathcal{K}^{4}\right]+\frac{1}{48}[\mathcal{K}]^{3}\left[\mathcal{K}^{2}\right]^{2}-\frac{1}{12}[\mathcal{K}]^{2}\left[\mathcal{K}^{2}\right] \\
& \cdot\left[\mathcal{K}^{3}\right]+\frac{1}{10}[\mathcal{K}]^{2}\left[\mathcal{K}^{5}\right]-\frac{1}{48}\left[\mathcal{K}^{2}\right]^{3}[\mathcal{K}]+\frac{1}{24}\left[\mathcal{K}^{2}\right] \\
& \cdot\left[\mathcal{K}^{5}\right]+\frac{1}{18}\left[\mathcal{K}^{3}\right]^{2}[\mathcal{K}]-\frac{1}{12}\left[\mathcal{K}^{3}\right]\left[\mathcal{K}^{4}\right] \\
& +\frac{1}{8}[\mathcal{K} 6][\mathcal{K}]+\frac{1}{7}\left[\mathcal{K}^{7}\right],
\end{aligned}
$$

where $\mathcal{K}$ is the matrix square root, namely $(\sqrt{A})_{\nu}^{\mu}(\sqrt{A})_{\lambda}^{\nu}=$ $A_{\lambda}^{\mu}$, and the rectangular brackets denote the traces, namely, $[\mathcal{K}]=\mathcal{K}_{v}^{\mu}$ and $\left[\mathcal{K}^{n}\right]=\left(K^{n}\right)_{v}^{\mu}[68]$. Based on the action, which is expressed as Eq. (13), the line element of a Ddimensional charged AdS black hole in dRGT massive gravity is expressed as

$\mathrm{d} s^{2}=-f(r) \mathrm{d} t^{2}+f(r)^{-1} \mathrm{~d} r^{2}+r^{2} \mathrm{~d} \Omega_{D-2}^{2}$,

where $\mathrm{d} \Omega_{D-2}^{2}=h_{i j} \mathrm{~d} x^{i} \mathrm{~d} x^{j}$ represents the line element of a $(D-2)$-dimensional space with constant curvature $(D-2)(D-3) k$, in which $k$ denotes the spatial curvature constant. When $k=0$, the horizon hypersurface of spacetime is flat. If the spatial curvature constant takes the value $k=1$, the geometric property of black hole horizon hypersurface has positive curvature, whereas it has negative curvature if $k=-1$. Considering reference metric $f_{\mu \nu}=\operatorname{diag}\left(0,0, c_{0}^{2} h_{i j}\right)$ and using the notation $d_{k}=D-k$, the metric function $f(r)$ can be expressed as follows:

$$
\begin{aligned}
f(r)= & k+c_{0}^{2} c_{2} m^{2}+\frac{r^{2}}{l^{2}}+\frac{c_{0} c_{1} m^{2}}{d_{2}} r-\frac{16 \pi M}{d_{2} V_{D-2} r^{D-3}} \\
& +\frac{d_{3} c_{0}^{3} c_{3} m^{2}}{r}+\frac{d_{3} d_{4} c_{0}^{4} c_{4} m^{2}}{r^{2}}+\frac{d_{3} d_{4} d_{5} c_{0}^{5} c_{5} m^{2}}{r^{2}} \\
& +\frac{d_{3} d_{4} d_{5} d_{6} c_{0}^{6} c_{6} m^{2}}{r^{2}}+\frac{d_{3} d_{4} d_{5} d_{6} d_{7} c_{0}^{7} c_{7} m^{2}}{r^{2}} \\
& +\frac{q^{2}}{2 d_{2} d_{3} r^{2 d_{3}}}+\cdots,
\end{aligned}
$$

where $V_{D-2}$ is the volume of space that is spanned by coordinates $x_{i}, M$ is the mass of the black hole [59], and $l$ represents the AdS radius. The electric field in a D-dimensional charged AdS black hole in dRGT massive gravity is $F_{t r}=1 / r^{d_{2}}$ and the electromagnetic potential becomes $A_{t}=-q /\left(d_{3} r^{d_{3}}\right)$. Constant $c_{0}$ satisfies $c_{0}>0$ and terms $c_{3} m^{2}$ and $c_{4} m^{2}$ vanish if $D<5$ and $D<6$, respectively. Term $c_{7} m^{2}$ only appears if $D \geq 9$; hence, for convenience, we only consider $n=7$ and $D \geq 9$ in this paper [69-71]. Based the geometric properties of the D-dimensional charged AdS black hole in dRGT massive gravity, its event horizon is located at $\left.f(r)\right|_{r=r_{H}}=0$. Moreover, one can obtain the line element of the D-dimensional Schwarzschild AdS black hole solution when $m \rightarrow 0$ and $q=0$. Based on the ghost-free dRGT theory and Eq. (14), the form of $f(r)$ in the above equation includes additional graviton terms, which rarely appear in previous works. Therefore, according to Eq. (14), one may obtain more physical information about higher dimensional nonlinear massive gravity.

Now, substituting the inverse tensors of the D-dimensional charged AdS black hole in dRGT massive gravity $g^{\mu \nu}$ into Eq. (12), the modified Hamilton-Jacobi equation can be rewritten as follows

$$
\begin{aligned}
& -f(r)^{-1}\left[\frac{\partial S}{\partial t}-\frac{e q}{(D-3) r^{(D-3)}}\right]^{2} \\
& +f(r)\left(\frac{\partial S}{\partial r}\right)^{2}+r^{-2}\left(\frac{\partial S}{\partial \Omega_{D-2}^{2}}\right)^{2} \\
& +2 \sigma m f(r)^{\frac{\beta+1}{2}}\left[-i \omega+i \frac{e q}{(D-3) r^{(D-3)}}\right]^{\beta+1} \\
& +m^{2}=0 .
\end{aligned}
$$

Considering the geometric properties of Eq. (15), the action can be represented as $S=-\omega t+W(r)+\Theta\left(\Omega_{D-2}\right)$. Hence, the radial part of Eq. (17) is

$$
\begin{aligned}
& -f(r)^{-1}\left[\omega+\frac{e q}{(D-3) r^{(D-3)}}\right]^{2} \\
& +f(r)\left[\frac{\partial W(r)}{\partial r}\right]^{2}+m^{2} \\
& +2 \sigma m f(r)^{\frac{\beta+1}{2}}\left[-i \omega+i \frac{e q}{(D-3) r^{(D-3)}}\right]^{\beta+1} \\
& +\lambda_{0}=0,
\end{aligned}
$$

and the nonradial part of the modified Hamilton-Jacobi equation becomes

$r^{-2}\left(\frac{\partial \Theta}{\partial \Omega_{D-2}^{2}}\right)^{2}-\lambda_{0}=0$ 
where $\lambda_{0}$ is a separation constant. For a spherically symmetric black hole, the nonradial part of the modified HamiltonJacobi equation does not correspond to the tunneling rate of the emitted particles; hence, here, we only consider Eq. (18). It should be noted that the result of Eq. (18) depends on the value of $\beta$. Therefore, the MDR effect has an important influence on the tunneling behavior of fermions; similar conclusions can be found in Refs. [38-40]. Hence, different values of $\beta$ lead to different tunneling rates. However, for convenience, we set $\beta=1$, which leads to the integral function

$$
\begin{aligned}
W_{ \pm}= & \pm \int \frac{d r}{f(r)} \\
& \cdot \sqrt{(1-2 \sigma m)\left(\omega-e A_{t}\right)^{2}-f(r)\left(m^{2}+\lambda_{0}\right)},
\end{aligned}
$$

where $+(-)$ represent the outgoing (incoming) solutions, respectively. The solution to the above integral on the event horizon is

$W_{ \pm}= \pm i \pi \frac{(1-m \sigma)}{f^{\prime}\left(r_{H}\right)}\left(\omega-\omega_{0}\right)$,

where $\omega_{0}=-(e q) /\left[(D-3) r_{H}^{D-3}\right]$. Based on the tunneling theory of black holes, the tunneling rate of fermions with half-integral spin at the event horizon is given by

$$
\begin{aligned}
\Gamma & =\exp \left[-\frac{2}{\hbar}\left(\operatorname{Im} W_{+}-\operatorname{Im} W_{-}\right)\right] \\
& =\exp \left[-\frac{4 \pi}{\hbar} \frac{(1-m \sigma)\left(\omega-\omega_{0}\right)}{f^{\prime}\left(r_{H}\right)}\right] .
\end{aligned}
$$

Since Eq. (22) is similar to the Boltzmann formula, the modified Hawking temperature of the D-dimensional charged AdS black hole in dRGT massive gravity becomes

$$
T_{H}=\frac{\hbar}{4 \pi r_{H}} \Xi(1+m \sigma)=T_{0}(1+m \sigma),
$$

with

$$
\begin{aligned}
\Xi= & d_{3} k+\frac{d_{1} r_{H}^{2}}{l^{2}}-\frac{q^{2}}{2 d_{3} r_{H}^{2 d_{3}}}+c_{0} c_{1} m^{2} r_{H}+d_{3} c_{2} c_{0}^{2} m^{2} \\
& +\frac{d_{3} d_{4} c_{3} c_{0}^{3} m^{2}}{r_{H}}+\frac{d_{3} d_{4} d_{5} c_{4} c_{0}^{4} m^{2}}{r_{H}^{2}}+\frac{d_{3} d_{4} d_{5} d_{6} c_{5} c_{0}^{5} m^{2}}{r_{H}^{2}} \\
& +\frac{d_{3} d_{4} d_{5} d_{6} d_{7} c_{6} c_{0}^{6} m^{2}}{r_{H}^{2}}+\frac{d_{3} d_{4} d_{5} d_{6} d_{7} d_{8} c_{7} c_{0}^{7} m^{2}}{r_{H}^{2}}+\cdots
\end{aligned}
$$

Besides, according to Ref. [68], we express the mass of the D-dimensional charged AdS black hole in dRGT massive gravity in terms of the event horizon as follows

$$
\begin{aligned}
M= & \frac{d_{2} V_{D-2} r_{H}^{d_{3}}}{16 \pi}\left[k+\frac{r_{H}^{2}}{l^{2}}+\frac{q^{2}}{2 d_{2} d_{3} r_{H}^{2 d_{2}}}+c_{0} c_{2} m^{2}\right. \\
& +\frac{c_{0} c_{1} m^{2} r_{H}}{d_{2}}+\frac{d_{3} c_{0}^{3} c_{3} m^{2}}{r_{H}}+\frac{d_{3} d_{4} c_{0}^{4} c_{4} m^{2}}{r_{H}^{2}} \\
& +\frac{d_{3} d_{4} d_{5} c_{0}^{5} c_{5} m^{2}}{r_{H}^{2}}+\frac{d_{3} d_{4} d_{5} d_{6} c_{0}^{6} c_{6} m^{2}}{r_{H}^{2}} \\
& \left.+\frac{d_{3} d_{4} d_{5} d_{6} d_{7} c_{0}^{7} c_{7} m^{2}}{r_{H}^{2}}+\cdots\right] .
\end{aligned}
$$

In Eq. (23), $T_{H}$ is the modified Hawking temperature of the D-dimensional charged AdS black hole in dRGT massive gravity, whereas $T_{0}$ is the original temperature.

Furthermore, by using Eq. (23) and the first law of black hole thermodynamics, namely, $\mathrm{d} S=\left(\mathrm{d} M-\Omega_{H} \mathrm{~d} J-\Phi \mathrm{d} Q\right)$ $/ T$ with the electromagnetic potential $\Omega_{H}$ and rotating potential $\Phi$, the modified entropy of the D-dimensional charged AdS black hole in dRGT massive gravity is expressed as

$$
\begin{aligned}
S_{H} & =\int \frac{\mathrm{d} M-\Phi \mathrm{d} Q}{(1+\sigma m) T_{0}} \\
& =S_{0}-m \sigma \int \mathrm{d} S_{0}+\mathcal{O}\left(\sigma^{2}\right),
\end{aligned}
$$

where $S_{0}=V_{D-2} r_{H}^{D-2} / 4$ is the original entropy of the Ddimensional charged AdS black hole in dRGT massive gravity. It is clear that both modified Hawking temperature $T_{H}$ and entropy $S_{H}$ not only are related to the properties of the Ddimensional charged AdS black hole in dRGT massive gravity but also depend on the coupling constant $\sigma$ and the mass of the emitted particles $m$. Our results more accurately describe the thermodynamic properties of charged AdS spacetime in dRGT gravity since the graviton terms are included. However, if $\sigma=0$, those modifications reduce to $T_{0}$ and $S_{0}$.

According to Eq. (25), the modified Hawking temperature is higher than the original temperature. Therefore, the effect of MDR can substantially enhance the evolution of the black hole. Hence, according to our result, MDR can accelerate the emission of particles from black holes. The emission rate of black holes is described by the Stefan-Boltzmann law. Therefore, it is interesting to study the effect of MDR on the Stefan-Boltzmann law, which is expressed as follows:

$\frac{d E}{d t}=\varrho_{S} A T^{4}$

where $E$ is the total energy of the black hole, $\varrho_{S}$ is the StefanBoltzmann constant, and $A$ and $T$ represent the area and temperature of the black hole, respectively. Now, by inserting Eq. (25) into Eq. (27), the Stefan-Boltzmann law can be rewritten as 


$$
\begin{aligned}
\left(\frac{d E}{d t}\right)_{\text {modified }} & =\sigma_{S} A T_{0}^{4}(1+m \sigma)^{4} \\
& =\left(\frac{d E}{d t}\right)_{\text {original }}(1+m \sigma)^{4}
\end{aligned}
$$

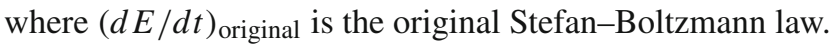
According to Eq. (28), the modified emission rate is higher than the original rate, which accords with a previous analysis of the Hawking temperature. The above results are obtained under the assumption $\beta=1$; if once $\beta$ takes another value, the final result will change accordingly.

\section{Discussion and conclusions}

In this paper, according to the MDR effect and the RaritaSchwinger equation, we constructed a deformed HamiltonJacobi equation in curved spacetime, which can be used to describe the kinetic characteristics of fermions with half-integral spin. Subsequently, the tunneling behavior of fermions with half-integral spin from a higher-dimensional AdS black hole in dRGT massive gravity is investigated via this modified Hamilton-Jacobi equation. It is found that if we fix $\beta=1$, the modified thermodynamic quantities not only are related to the properties of spacetime of the higher dimensional charged AdS black hole in dRGT massive gravity but also depend on the coupling constant $\sigma$ and the mass of the emitted particles $m$. Meanwhile, we calculated the modified emission rate by using Stefan-Boltzmann's law. Our results demonstrate that the modified values are higher than the original values; hence, the effect of MDR can substantially enhance the evolution of the black hole. Notably, based on ghost-free dRGT theory, the graviton terms, namely, $\mathcal{U}_{i}$ can be extended to higher dimensions, which leads to the form of $f(r)$ in Eq. (16), which differs from those in previous works. Therefore, one may obtain more physical information about higher dimensional nonlinear massive gravity via our results. In addition, this work demonstrates that the effect of MDR has a substantial influence on the deformed HamiltonJacobi equation: the modified tunneling rate of fermions, the Hawking temperature, the entropy and the Stefan-Boltzmann law depend on the value of parameter $\beta$. It is believed that interesting and meaningful results can be obtained from this finding, which we will discuss in detail in future works.

\begin{abstract}
Acknowledgements The authors thank Prof. Kai Lin and the anonymous referees for helpful suggestions and enlightening comments, which helped to improve the quality of this paper. This work is supported in part by the National Natural Science Foundation of China (Grant nos. 11847048, 11703018 and 11573022) and the Fundamental Research Funds of China West Normal University (Grant nos. 17E093, 17YC518, 18Q067, cxcy2018235 and cxcy2018227).
\end{abstract}

Data Availability Statement This manuscript has no associated data or the data will not be deposited [Authors' comment: This article is composed of mathematical and physical deductions, without specific data.]

Open Access This article is distributed under the terms of the Creative Commons Attribution 4.0 International License (http://creativecomm ons.org/licenses/by/4.0/), which permits unrestricted use, distribution, and reproduction in any medium, provided you give appropriate credit to the original author(s) and the source, provide a link to the Creative Commons license, and indicate if changes were made. Funded by SCOAP ${ }^{3}$.

\section{References}

1. S.M. Carroll, Spacetime and Geometry: An Introduction to General Relativity (Addison-Wesley, San Fracnisco, 2004)

2. S.W. Hawking, Nature 248, 30 (1974). https://doi.org/10.1038/ 248030a0

3. S.W. Hawking, Commun. Math. Phys. 4(3), 199 (1975). https:// doi.org/10.1007/BF02345020

4. T. Damour, R. Runi, Phys. Rev. D 14, 332 (1976). https://doi.org/ 10.1103/PhysRevD.14.332

5. S. Sannan, Gen. Relativ. Gravit. 20, 239 (1988). https://doi.org/10. 1007/BF00759183

6. P. Kraus, F. Wilczek, Nucl. Phys. B 433, 403 (1995). https://doi. org/10.1016/0550-3213(94)00411-7

7. M.K. Parikh, F. Wilczek, Phys. Rev. Lett. 85, 5042 (2000). https:// doi.org/10.1103/PhysRevLett.85.5042

8. K. Srinivasan, T. Padmanabhan, Phys. Rev. D 60, 24007 (1999). https://doi.org/10.1103/PhysRevD.60.024007

9. R. Kerner, R.B. Mann, Class. Quant. Grav. 25, 095014 (2008). https://doi.org/10.1088/0264-9381/25/9/095014

10. R. Kerner, R.B. Mann, Phys. Lett. B 665, 277 (2008). https://doi. org/10.1016/j.physletb.2008.06.012

11. K. Lin, S.Z. Yang, Chin. Phys. B 20, 110403 (2011). https://doi. org/10.1088/1674-1056/20/11/110403

12. Z.W. Feng, H.L. Li, X.T. Zu, S.Z. Yang, Eur. Phys. J. C 76, 212 (2016). https://doi.org/10.1140/epjc/s10052-016-4057-1

13. J.Y. Zhang, Z. Zhao, Phys. Lett. B 638, 110 (2006). https://doi.org/ 10.1016/j.physletb.2006.05.059

14. B. Chatterjee, A. Ghosh, P. Mitra, Phys. Lett. B 661, 307 (2008). https://doi.org/10.1016/j.physletb.2008.02.034

15. K. Lin, S.Z. Yang, Phys. Rev. D 79, 064035 (2009). https://doi.org/ 10.1103/PhysRevD.79.064035

16. K. Lin, S.Z. Yang, Phys. Lett. B 674, 127 (2009). https://doi.org/ 10.1016/j.physletb.2009.02.055

17. H.L. Li, R. Lin, L.Y. Cheng, Europhys. Lett. 98, 30002 (2012). https://doi.org/10.1209/0295-5075/98/30002

18. G.-R. Chen, Y.-C. Huang, Gen. Relativ. Gravit. 47, 57 (2015). https://doi.org/10.1007/s10714-015-1897-1

19. I. Sakalli, H. Gursel, Eur. Phys. J. C 76, 318 (2016). https://doi. org/10.1140/epjc/s10052-016-4158-X

20. Z.W. Feng, Y. Chen, X.T.Zu, Astrophys. Space Sci. 359, 48 (2015). https://doi.org/10.1007/s10509-015-2498-x

21. X.-M. Kuang, J. Saavedra, A. Övgün, Eur. Phys. J. C 77, 613 (2017). https://doi.org/10.1140/epjc/s10052-017-5191-0

22. Z.W. Feng, Int. J. Theor. Phys. 57, 931 (2018). https://doi.org/10. 1007/s10773-017-3625-y

23. P. Chen, Y.C. Ong, D.-H. Yeom, Phys. Rep. 603, 1 (2015). https:// doi.org/10.1016/j.physrep.2015.10.007

24. K. Konishi, G. Paffuti, P. Provero, Phys. Lett. B 234, 276 (1990). https://doi.org/10.1016/0370-2693(90)91927-4

25. M. Maggiore, Phys. Lett. B 319, 83 (1993). https://doi.org/10. 1016/0370-2693(93)90785-G 
26. L.J. Garay, Int. J. Mod. Phys. A 10, 145 (1995). https://doi.org/10. 1142/S0217751X95000085

27. G. Amelino-Camelia, Int. J. Mod. Phys. D 11, 35 (2002). https:// doi.org/10.1142/S0218271802001330

28. G. Amelino-Camelia, New J. Phys. 6, 188 (2004). https://doi.org/ $10.1088 / 1367-2630 / 6 / 1 / 188$

29. A.F. Ali, Phys. Rev. D 89, 104040 (2014). https://doi.org/10.1103/ PhysRevD.89.104040

30. A.F. Ali, M. Faizal, M.M. Khalil, J. High Energy Phys. 1412, 159 (2014). https://doi.org/10.1007/JHEP12(2014)159

31. A.F. Ali, M. Faizal, M.M. Khalil, Nucl. Phys. B 894, 341 (2015). https://doi.org/10.1016/j.nuclphysb.2015.03.014

32. S.H. Hendi, M. Faizal, Phys. Rev. D 92, 044027 (2015). https:// doi.org/10.1103/PhysRevD.92.044027

33. A.N. Tawfik, A.M. Diab, Int. J. Mod. Phys. A 30, 1550059 (2015). https://doi.org/10.1142/S0217751X15500591

34. C.B. Hinojosa, J. López-Sarrión, Phys. Lett. B 749, 431 (2015). https://doi.org/10.1016/j.physletb.2015.08.006

35. P. Wang, H. Yang, S. Ying, Eur. Phys. J. C 76, 27 (2016). https:// doi.org/10.1140/epjc/s10052-015-3858-y

36. Y. Gim, W. Kim, Eur. Phys. J. C 76, 166 (2016). https://doi.org/10. 1140/epjc/s10052-016-4025-9

37. Z.W. Feng, S.Z. Yang, Phys. Lett. B 772, 737 (2017). https://doi. org/10.1016/j.physletb.2017.07.057

38. S.I. Kruglov, Phys. Lett. B 718, 228 (2012). https://doi.org/10. 1016/j.physletb.2012.10.037

39. S.I. Kruglov, Mod. Phys. Lett. A 28, 1350014 (2013). https://doi. org/10.1142/S0217732313500144

40. S.Z. Yang, K. Lin, J. Lin, Q.Q. Jiang, Adv. High Energy Phys. 2016, 7058764 (2016). https://doi.org/10.1155/2016/7058764

41. S.Z. Yang, K. Lin, Sci. China Phys. Mech. 49, 019503 (2019). https://doi.org/10.1360/SSPMA2018-00307

42. W. Rarita, J. Schwinger, Phys. Rev. 60, 61 (1941). https://doi.org/ 10.1103/PhysRev.60.61

43. D. Colladay, V.A. Kostelecký, Phys. Rev. D 55, 6760 (1997). https:// doi.org/10.1103/PhysRevD.55.6760

44. V.A. Kostelecký, M. Mewes, Phys. Rev. D 88, 096006 (2013). https://doi.org/10.1103/PhysRevD.88.096006

45. A.F. Ali, M. Faizal, M.M. Khalil, J. High Energy Phys. 12, 159 (2014). https://doi.org/10.1007/JHEP12(2014)159

46. D.Y. Chen, H.W. Wu, H.T. Yang, J. Cosmol. Astropart. Phys. 03, 036 (2014). https://doi.org/10.1088/1475-7516/2014/03/036

47. K. Nozari, S. Saghafi, A.D. Kamali, Astrophys. Space Sci. 357, 140 (2015). https://doi.org/10.1007/s10509-015-2371-y

48. B. Mu, P. Wang, H. Yang, Adv. High Energy Phys. 2015, 898916 (2015). https://doi.org/10.1155/2015/898916
49. X.-X. Zeng, Y. Chen, Gen. Relativ. Gravit. 47, 47 (2015). https:// doi.org/10.1007/s10714-015-1890-8

50. X.-X. Zeng, X.-M. Liu, L.-F. Li, Eur. Phys. J. C 76, 616 (2016). https://doi.org/10.1140/epjc/s10052-016-4463-4

51. X.-X. Zeng, L.-F. Li, Phys. Lett. B 764, 100 (2017). https://doi. org/10.1016/j.physletb.2016.11.017

52. S.H. Hendi, B.E. Panah, S. Panahiyan, Phys. Lett. B 769, 191 (2017). https://doi.org/10.1016/j.physletb.2017.03.051

53. I. Sakalli, A. Övgün, K. Jusufi, Astrophys. Space Sci. 361, 330 (2016). https://doi.org/10.1007/s10509-016-2922-x

54. S.H. Hendi, A. Dehghani, M. Faizal, Nucl. Phys. B 914, 117 (2017). https://doi.org/10.1016/j.nuclphysb.2016.10.022

55. E. Marsch, Eur. Phys. J. Plus 132, 188 (2017). https://doi.org/10. 1140/epjp/i2017-11460-6

56. R. Mandal, S. Bhattacharyya, S. Gangopadhyay, Gen. Relativ. Gravit. 50, 143 (2018). https://doi.org/10.1007/ s10714-018-2468-z

57. H.-L. Li, Z.-W. Feng, S.-Z. Yang, X.-T. Zu, Eur. Phys. J. C 78, 768 (2018). https://doi.org/10.1140/epjc/s10052-018-6252-8

58. Y.-F. Cai, D.A. Easson, C. Gao, E.N. Saridakis, Phys. Rev. D 87, 06400 (2013). https://doi.org/10.1103/PhysRevD.87.064001

59. D. Vegh, arXiv:1301.0537 [hep-th]

60. R.G. Cai, Y.P. Hu, Q.Y. Pan, Y.L. Zhang, Phys. Rev. D 91, 024032 (2015). https://doi.org/10.1103/PhysRevD.91.024032

61. S.H. Hendi, B.E. Panah, S. Panahiyan, J. High Energy Phys. 2015, 157 (2015). https://doi.org/10.1007/JHEP11(2015)157

62. S.H. Hendi, S. Panahiyan, B.E. Panah, J. High Energy Phys. 2016, 129 (2016). https://doi.org/10.1007/JHEP01(2016)129

63. X.-H. Feng, H. Huang, S.-L. Li, H. Lu, H. Wei, arXiv:1807.01720 [hep-th]

64. D.-C. Zou, Y. Liu, R. Yue, Eur. Phys. J. C 77, 365 (2017). https:// doi.org/10.1140/epjc/s10052-017-4937-Z

65. T.Q. Do, Phys. Rev. D 93, 104003 (2016). https://doi.org/10.1103/ PhysRevD.93.104003

66. T.Q. Do, Phys. Rev. D 94, 044022 (2016). https://doi.org/10.1103/ PhysRevD.94.044022

67. T.Q. Do, J. Phys. Conf. Ser. 865, 012001 (2017). https://doi.org/ 10.1088/1742-6596/865/1/012001

68. D.-C. Zou, R. Yue, M. Zhang, Eur. Phys. J. C 77, 256 (2017). https://doi.org/10.1140/epjc/s10052-017-4822-9

69. C. de Rham, G. Gabadadze, Phys. Rev. D 82, 044020 (2010). https://doi.org/10.1103/PhysRevD.82.044020

70. C. de Rham, G. Gabadadze, A.J. Tolley, Phys. Rev. Lett. 106, 231101 (2011). https://doi.org/10.1103/PhysRevLett.106.231101

71. B. Liu, Z.-Y. Yang, R.-H. Yue, arXiv: 1810.07885 